\title{
Method to determine thermoelastic material properties of constituent and copper-patterned layers of multilayer printed circuit boards
}

\author{
J. F. J. Veldhuijzen van Zanten ${ }^{1} \cdot$ G. A. Schuerink ${ }^{2} \cdot$ A. H. J. Tullemans ${ }^{2} \cdot$ R. Legtenberg ${ }^{2} \cdot$ Wessel W. Wits $^{1}$ (i)
}

Received: 9 October 2017 / Accepted: 18 December 2017 / Published online: 4 January 2018

(c) The Author(s) 2018. This article is an open access publication

\begin{abstract}
Due to the ongoing component miniaturization and integration in the electronics industry, there is a need for asymmetric lay-ups for printed circuit boards (PCBs), especially in the case of complex boards that house both analog and digital circuits. This paper focuses on the contribution of the constituent layers that make up the PCB to the board's macroscopic multilayer properties in terms of stiffness and coefficient of thermal expansion (CTE). The thermoelastic material properties for constituents, like cured prepreg and laminate layers, have been determined. Using classical laminate theory, individual layer properties are assembled to the macroscopic level and compared to fabricated multilayer boards. Following this approach, the contribution of various copper features on constituent layers can de deduced. The experiments show that properties of cured prepreg, taking $z$-direction expansion into account, and laminate layers are dependent on the type of fiberglass reinforcement and the fiber volume fraction. Depending on these properties, the Young's modulus and CTE varies from 11 to $31 \mathrm{GPa}$ and from 10 to $28 \mathrm{ppm} / \mathrm{K}$, respectively. Datasheet values deviate significantly from these results as they do not take the fiber volume fraction into account. By alternating the measurement directions, the experiments have also shown that the fiberglass reinforcement plays a dominant role in determining macroscopic multilayer board properties. The multilayer board follows iso-strain conditions. Therefore, the material properties depend linearly on the copper volume fraction and follow the rule of mixtures independent of the type of copper patterning. Overall, the presented model and method to determine material properties increase the accuracy for predicting multilayer board behavior and offers the possibility to design and predict bow and twist behavior of PCBs with asymmetric lay-ups.
\end{abstract}

\section{Introduction}

To meet the future demands in Radio Frequent (RF) and mixed-signal (i.e. analog and digital) Printed Circuit Boards (PCBs), the density of electronic components and number of integrated functionalities within the PCB must increase [1-3]. Due to the electrical complexity (component mix, RF and digital sides) and resulting design constraints, a deviation from the traditional symmetric board lay-up may be required. To get better performances of the electrical circuit, more beneficial di-electrical properties can be achieved by asymmetric lay-ups [4]. Hence, a shift to asymmetric boards may be preferred to create better performing PCBs.

Wessel W. Wits

w.w.wits@utwente.nl

1 Faculty of Engineering Technology, University of Twente, P.O. Box 217, 7500 AE Enschede, The Netherlands

2 Thales Nederland B.V., P.O. Box 42, 7550 GD Hengelo, The Netherlands
An asymmetric board is however often one of the main root causes of PCB deformation in bow and twist modes due to an unbalance of the board's stiffness and the load situation relative to the board's center line [5]. The load situation is a result of the differences in Coefficient of Thermal Expansion (CTE) and Young's modulus between the individual layers that make up the board. Other root causes capable of causing unbalanced load situations are process-induced phenomena, as e.g. press and bonding process steps.

Earlier work on this topic was presented by Schuerink et al. [6] in 2013. They have presented an analytical method to predict PCB deformation induced by its manufacturing process steps. This method followed classical laminate theory (CLT) as a basis, tracking internal stresses and includes the results of subsequent production steps, such as bonding, multilayer press cycles and patterning processes by defining an equivalent thermal history. However, for this model it is imperative to have accurate material properties of the constituent PCB materials. Therefore, the focus of this study is on determining the in-plane thermoelastic material 
properties of the composite layers. More specifically, the CTE and Young's modulus are determined for laminates, cured prepregs and copper-patterned layers (i.e. copper features in-between which prepreg flows during the bonding process).

Literature data on the material properties of fabricated constituent layers within a PCB-being a combination of etched copper features filled with epoxy resin-is scarce to non-existing. It is difficult to determine the as-built material properties of specific layers since they are always accompanied by one or more surrounding layers. Next to the surrounding layers, it has also been shown that a layer's CTE and Young's modulus are significantly influenced by the copper trace percentage of that specific layer [7]. In this study, a reverse engineering approach is employed to deduce the properties of constituent PCB layers. First, the material properties of a complete multilayer PCB are characterized through ThermoMechanical Analysis (TMA) and tensile stress testing. Second, the material properties of individual fiberglass reinforced composite layers are derived using CLT; in particular a composites modeling software tool, U20MM, is used [8]. Third, the individual contribution of each layer is deduced following rules of mixtures to model as-built material properties for the constituent copperpatterned layers. The resulting material properties serve as input to predict the bow and twist of PCBs after the bonding process step, e.g. by following the aforementioned analytical model of Schuerink et al. [6].

\section{Multilayer PCB material composition}

Multilayer PCBs are a stacked combination of laminates and copper layers, with or without copper features, bonded together by prepregs $[9,10]$. Interconnecting vias are usually drilled after bonding to connect various layers electrically. The prepregs are usually thin layers, $50-180 \mu \mathrm{m}$, while laminates may range from 50 up to $3000 \mu \mathrm{m}$. Both laminates and prepregs used in this study are thermosetting polymer composites consisting of a matrix material that binds its continuous fiberglass weave reinforcement. The degree of cure of the matrix material is what distinguishes prepregs from laminates. Prepregs are in the so-called B-stage of curing, implying that they are partially cured. By exposing the B-stage prepreg material to elevated temperatures and pressure for a predetermined time, it becomes low viscous once more before reaching the fully cured stage: the $\mathrm{C}$-stage [11]. The heat provided by the hot press is required for initiating and maintaining the chemical reactions in the resin, which cause the desired changes in molecular structure. High pressure makes sure the resin flows between the copper features and exposed laminates [12]. As the crosslinking reaction completes, a high degree of cure that bonds the layers together is achieved. Phase change, accompanied by a change of material properties, occurs during the curing process, which is typically described in a Time-TemperatureTransition (TTT) cure diagram that covers both effects of gelation and vitrification [13]. After curing, the prepreg can be considered and treated as a laminate.

\subsection{Resin matrix material: FR-4 epoxy}

Various types of material are used for the thermosetting resin matrix. Each supplier has its own proprietary recipe for the matrix material. Hence exact compositions are unknown. The most common materials are epoxy based and graded Flame Retardant (denoted by FR-4). They have a low operating temperature, are relatively low cost, and feature high process-ability and decent di-electric material properties. Epoxy resins have a glass transition temperature $\left(T_{g}\right)$ around $150-180{ }^{\circ} \mathrm{C}[14]$.

\subsection{Fiberglass reinforcements}

Laminates and prepregs are reinforced by one or multiple plain weaves: continuous fiberglass clothes made of electrical grade glass (E-glass). The resin binds the fiberglass weaves together to form the prepreg or laminate composite. The fiberglass weaves come in various patterns and are chosen based on the structural (e.g. thickness) and curing requirements, such as the amount of resin flow.

Commonly used weaves have fibers in the warp and fill directions; therefore, they can exhibit orthotropic properties at the macroscopic level. Warp is designated in the direction of the length of the roll, the 'machine direction', whereas fill is designated in the direction of the yarns, which 'fill in' from side to side during the weaving process of the cloth. The warp direction is usually associated with the principle $x$-direction of the laminate or prepreg (i.e. parallel to the longest side). Figure 1 shows and Table 1 lists the composition of a number of common plain weaves used in the electronics industry. Due to the variation of fabric count in warp and fill directions, the material properties per type of fiberglass cloth will differ in warp and fill directions as well. Various laminate and prepreg thicknesses are available, in which the cloth remains the same but the amount of resin varies. The laminate and prepreg thicknesses after production will vary, and hence so will the glass-to-resin ratio. Both properties are important to know in order to determine the influence of the individual fiberglass reinforced composite layers [15] and is subject to investigation in this study.

Referring to Table 1, the 0106 and 1080 cloth styles are usually used for thin laminates and prepregs due to their fine weave. They have an expanded weave pattern that is advantageous for resin flow during the bonding process. The $211 \times$ cloth styles are used in medium thickness applications and 
Fig. 1 Commonly used glass fiber plain weaves in the electronics industry; image taken from [16]

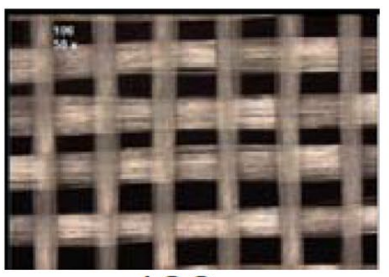

106

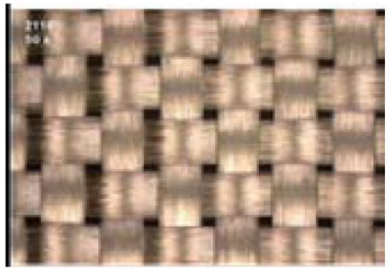

2116
Table 1 Commonly used glass fiber plain weaves in the electronics industry [15]

\begin{tabular}{llll}
\hline Cloth style & $\begin{array}{l}\text { Fabric count } \\
\text { Warp } \times \text { Fill }\left(\mathrm{cm}^{-1}\right)\end{array}$ & $\begin{array}{l}\text { Nominal } \\
\text { weight }\left(\mathrm{g} / \mathrm{m}^{2}\right)\end{array}$ & $\begin{array}{l}\text { Glass fiber } \\
\text { thickness } \\
(\mathrm{mm})\end{array}$ \\
\hline 0106 & $22.0 \times 22.0$ & 24.4 & 0.033 \\
1080 & $23.6 \times 18.5$ & 46.8 & 0.053 \\
1501 & $18.1 \times 17.7$ & 165.0 & 0.140 \\
1652 & $20.5 \times 20.5$ & 138.3 & 0.114 \\
1674 & $15.7 \times 12.6$ & 96.6 & 0.097 \\
2113 & $23.6 \times 22.0$ & 78.0 & 0.079 \\
2114 & $22.0 \times 18.9$ & 90.9 & 0.084 \\
2116 & $23.6 \times 22.8$ & 104.0 & 0.094 \\
7628 & $17.3 \times 12.2$ & 204.4 & 0.173 \\
\hline
\end{tabular}

normal resin flow requirements. An example of the usage of the 2114 cloth style and a schematic representation of the cross section are shown in Fig. 2. A cross section of the 2114 cloth style laminate in fill direction is shown. The layer is laminated with copper on each side (in grey) and two thin 0106 cloth style prepregs on the outside top and bottom. The figure clearly shows a thicker fiberglass cloth for the 2114

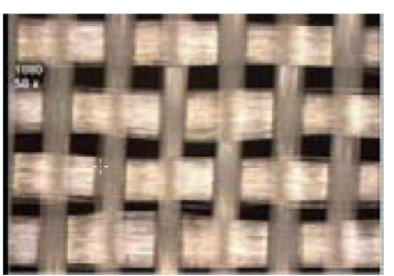

1080

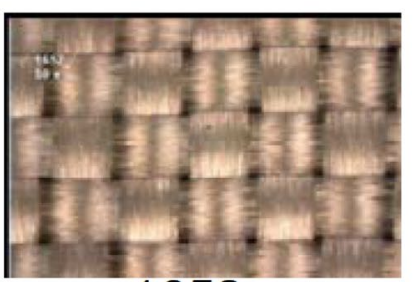

1652

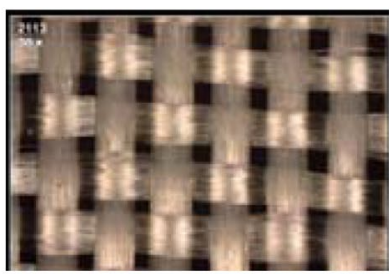

2113

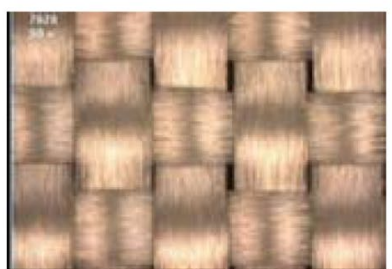

7628 cloth style laminate in the middle. Also, the fibers in warp and fill directions are visible. The thickest weaves, usually the $7 \times \times \times$ cloth styles, are used for the thickest laminates and prepregs, and prevent resin flow the most. A disadvantage of the $7 \times \times \times$ cloth styles is the rough surface finish, which can be compensated for by adding a smoother and finer cloth, like the 0106 and 1080 cloth styles, on top of the surface.

Laminates and prepregs with a specific fiberglass reinforcement, for instance the 2114 cloth style, are available in multiple predetermined thicknesses. The volume of the fiberglass remains the same while the amount of resin accounts for the variation in thickness. A variation in fiberglass volume fraction $\left(V_{f}\right)$ per type of product is the result. This volume fraction has an effect on the combined material properties of the composite [15].

The material properties of the E-glass fiber, the epoxy resin (below and above $T_{g}$ ) and bulk copper are tabulated in Table 2. Values are gathered from various sources as referenced. The E-glass fiber is a dense and stiff reinforcement with a low CTE while the epoxy resin has a low stiffness and high CTE at about half the density of E-glass. Above $T_{g}$ the stiffness of the epoxy resin drops an order of 100 while its CTE increases about fourfold. The Poisson's ratio $(\nu)$

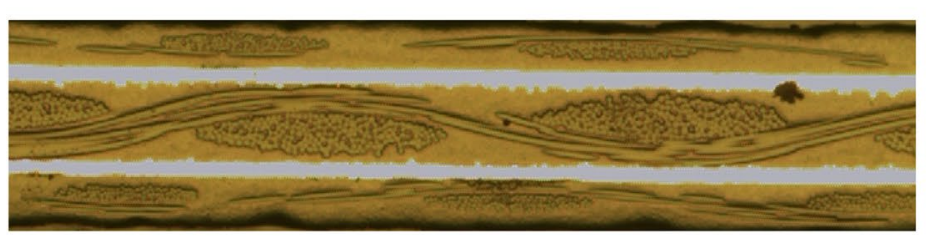

(a)

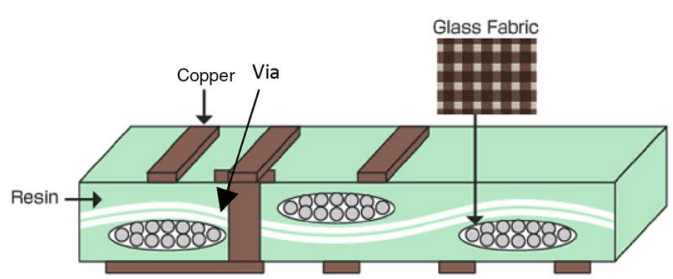

(b)

Fig. 2 Laminate cross section in fill direction. a Fiberglass 2114 cloth style laminate in the middle surrounded by two copper layers (in grey) and two fiberglass 0106 cloth style prepregs on the outside. b Schematic representation of a laminate cross section including an interconnecting via 
Table 2 Material properties of E-glass fiber [17], epoxy resin [17] and bulk copper [6]

\begin{tabular}{llllll}
\hline Material property & Symbol (unit) & E-glass fiber & \multicolumn{2}{l}{ Epoxy resin } & \multirow{2}{*}{ Bulk copper } \\
\cline { 5 - 6 } & & & Below $T_{g}$ & Above $T_{g}$ & \\
\hline Young's modulus & $E(\mathrm{GPa})$ & 72.3 & 3.44 & 0.07 & 115 \\
CTE & $\alpha(\mathrm{ppm} / \mathrm{K})$ & 5.1 & 58 & 197 & 17 \\
Poisson's ratio & $\nu(-)$ & 0.22 & 0.33 & 0.33 & 0.33 \\
Density & $\rho\left(\mathrm{g} / \mathrm{cm}^{3}\right)$ & 2.59 & 1.4 & - & 8.96 \\
\hline
\end{tabular}

of the epoxy resin is not significantly affected by the glass transition. The E-glass fiber and bulk copper properties are considered temperature independent around the resin's glass transition temperature.

\section{Orthotropic material modeling}

The analysis of composite materials can be done, following CLT, on several levels of complexity, from the micromechanics level up to the macro-mechanics level, as indicated in Fig. 3, with mini and meso levels in between [8]. At the micro-mechanics level, a material is modeled as a material reinforced with a plain weave continuous fiberglass cloth that is elastically loaded in the fiber direction. The model considers iso-strain, implying that the strain of both the resin and fibers are equal. Thermoelastic material properties can be determined for such layers. On the other hand, at the macro-mechanics level the behavior of the whole composite is studied. The material is presumed to be homogeneous, and the effects of the constituent materials are detected only as averaged apparent macroscopic properties of the composite [18]. This implies that the layers are treated as homogeneous layers with their properties determined by the model at the micro-mechanics level. Throughout this study, both models are used interchangeably. Micro-mechanic models are used to determine the thermoelastic properties of individual layers such as copper track and plane layers, and fiberglass reinforced layers. Macroscopic models are used to predict the deformation of a PCB consisting of various layers through applying CLT.

\subsection{Micro-mechanics of composites}

Weaves do not have a homogenous fiber distribution. Fibers are concentrated in the yarns and these are undulated in the weave. This implies that CLT cannot be applied directly to find the thermoelastic properties of composites build from woven fabric reinforced composite materials [8].

Through a micro-mechanics modeler, a utility tool to compute thermoelastic properties of woven fabric composites, meso-level mechanics can be predicted. The modeler extends CLT to account for the inhomogeneous fiber distribution in the weave and the undulation of the yarns. A so-called top-downbottom-up method is employed to predict the thermoelastic properties of a fabric reinforced composite, as indicated by the arrows in Fig. 3. This method consists of two parts: (1) the geometrical sub-division of the composite, the geometrical
Fig. 3 Different complexity levels of mechanics material models [8]
TOP

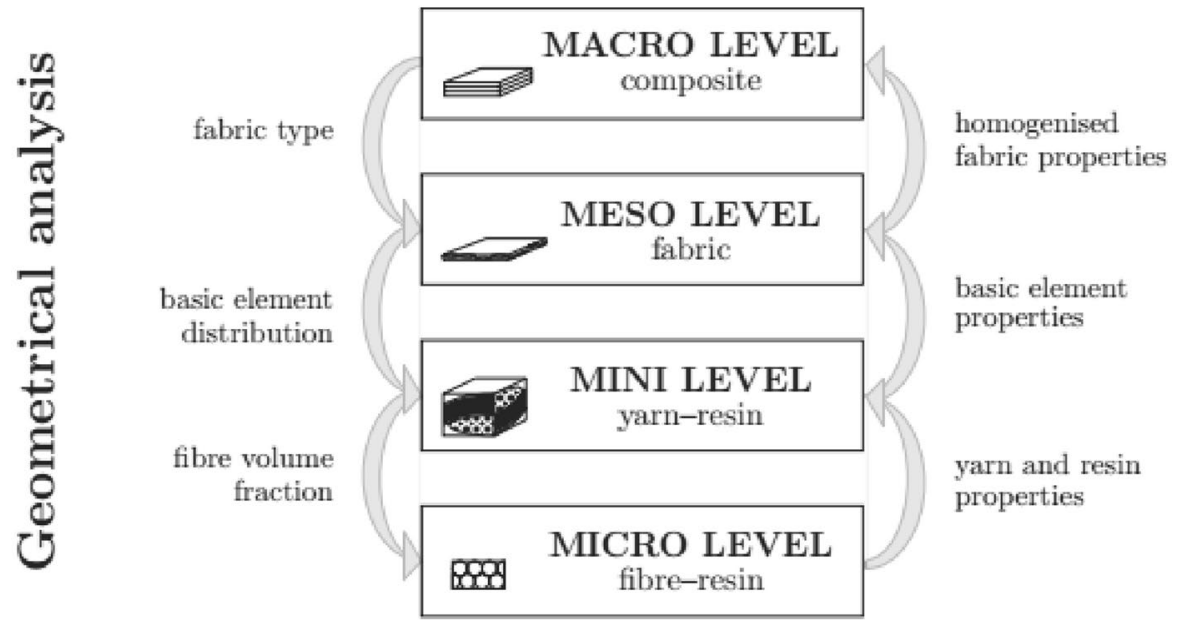

BOTTOM 
analysis, and (2) the subsequent rebuilding of the weave with its thermoelastic properties, the thermoelastic analysis [8]. In this study, the micro-mechanics modeler tool U20MM [19] is used to determine the thermoelastic properties for both the prepregs and laminates.

\subsection{Copper-patterned layers}

Copper layers have features that are determined by the design of the electrical circuit. During the curing process, resin flows between the copper features in the interior of the PCB and resides there. The combination of anisotropic copper features and prepreg resin in-between creates a layer with anisotropic material properties at the macroscopic level of the layer.

In practice, most copper-patterned layers are anisotropic. Three generic modeling techniques are available however to determine the layer properties of various types of copper patterns and stem from composite material science [20]. They are based on an equivalent stiffness determined by rules of mixture and include geometric information of the copper patterning and the copper percentage. The first modeling technique considers the parallel stiffness of a combination of the two materials, as shown in the pattern of Fig. 4(a), and is defined by:

$E_{p}=E_{1} V_{1}+E_{2} V_{2} \Rightarrow E_{p}=E_{1} V_{1}+\left(1-V_{1}\right) E_{2}$

where $E_{p}$ is the Young's modulus of the composite in longitudinal parallel direction, $V$ the volume fraction of the material with subscripts 1 and 2 referring to materials 1 (black) and 2 (white), respectively. Naturally, the sum of both volume fractions must equal unity. Equation 1 is based on the assumption of iso-strain. The linear CTE in longitudinal direction of a combination of two materials can be calculated by:

$\alpha_{p}=\frac{\alpha_{1} E_{1} V_{1}+\alpha_{2} E_{2} V_{2}}{E_{1} V_{1}+E_{2} V_{2}}$

where $\alpha_{p}$ is the linear CTE of a composite in which materials 1 and 2 reside in parallel. Similar to Eq. 1 this is assuming iso-strain conditions.

Fig. 4 Three considered types of copper patterning. a Parallel pattern (iso-strain). b Serial pattern (iso-stress). c Advanced pattern
The serial stiffness of a combination of two materials, as seen in the pattern of Fig. 4(b), is defined by:

$E_{s}=\frac{E_{1} E_{2}}{\left(1-V_{1}\right) E_{1}+E_{2} V_{1}}$

where $E_{s}$ is the Young's modulus of the composite with a serial pattern. Equation 3 is based on the assumption of isostress. Finally, for an advanced material pattern, as shown in the pattern of Fig. 4(c), a combination of parallel and serial stiffnesses is used:

$E_{a}=\left(1-\sqrt{V_{1}}\right) E_{2}+\frac{\sqrt{V_{1}} E_{1} E_{2}}{V_{1} E_{2}+\sqrt{V_{1}}\left(1-\sqrt{V_{1}}\right) E_{1}}$

where $E_{a}$ is the Young's modulus of the advanced pattern for materials 1 and 2 .

\section{Determining micro-mechanic material properties}

\subsection{Approach}

To determine the material properties of copper-patterned layers, a specially designed test board was produced that features various types of copper patterns. For each pattern, the material properties at the micro-mechanic level are determined. Figure 5 shows the layout and geometry of the copper-patterned layer of the board. The board is divided in five separated areas that are mutually independent. Three areas are covered by copper patterns, one area has a full copper layer and one area is free of copper. The shape of the patterns are motivated by Fig. 4 and Eqs. 1-4. The $X$-pattern and $Y$-pattern copper patterns consist of continuous copper tracks in their respective directions. The $X$-pattern copper has $200 \mu \mathrm{m}$ copper tracks, spaced by $400 \mu \mathrm{m}$ of resin. The $Y$-pattern copper has $200 \mu \mathrm{m}$ copper tracks, spaced by $200 \mu \mathrm{m}$ of resin. The advanced pattern has $200 \mu \mathrm{m}$ copper

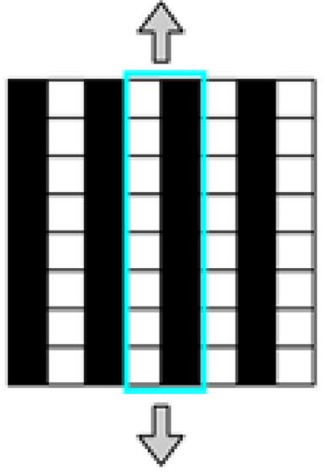

(a)

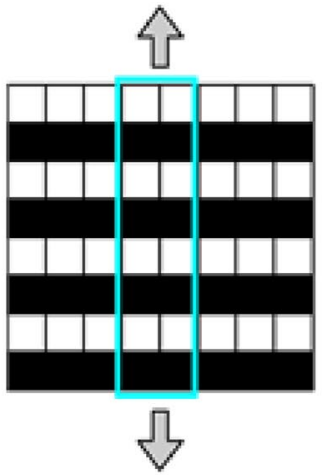

(b)

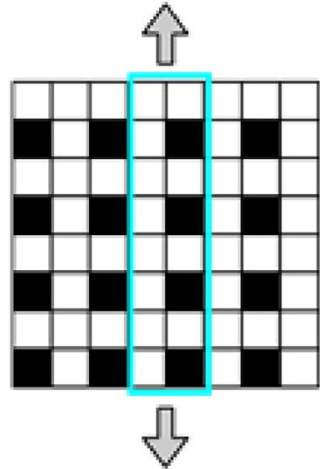

(c) 


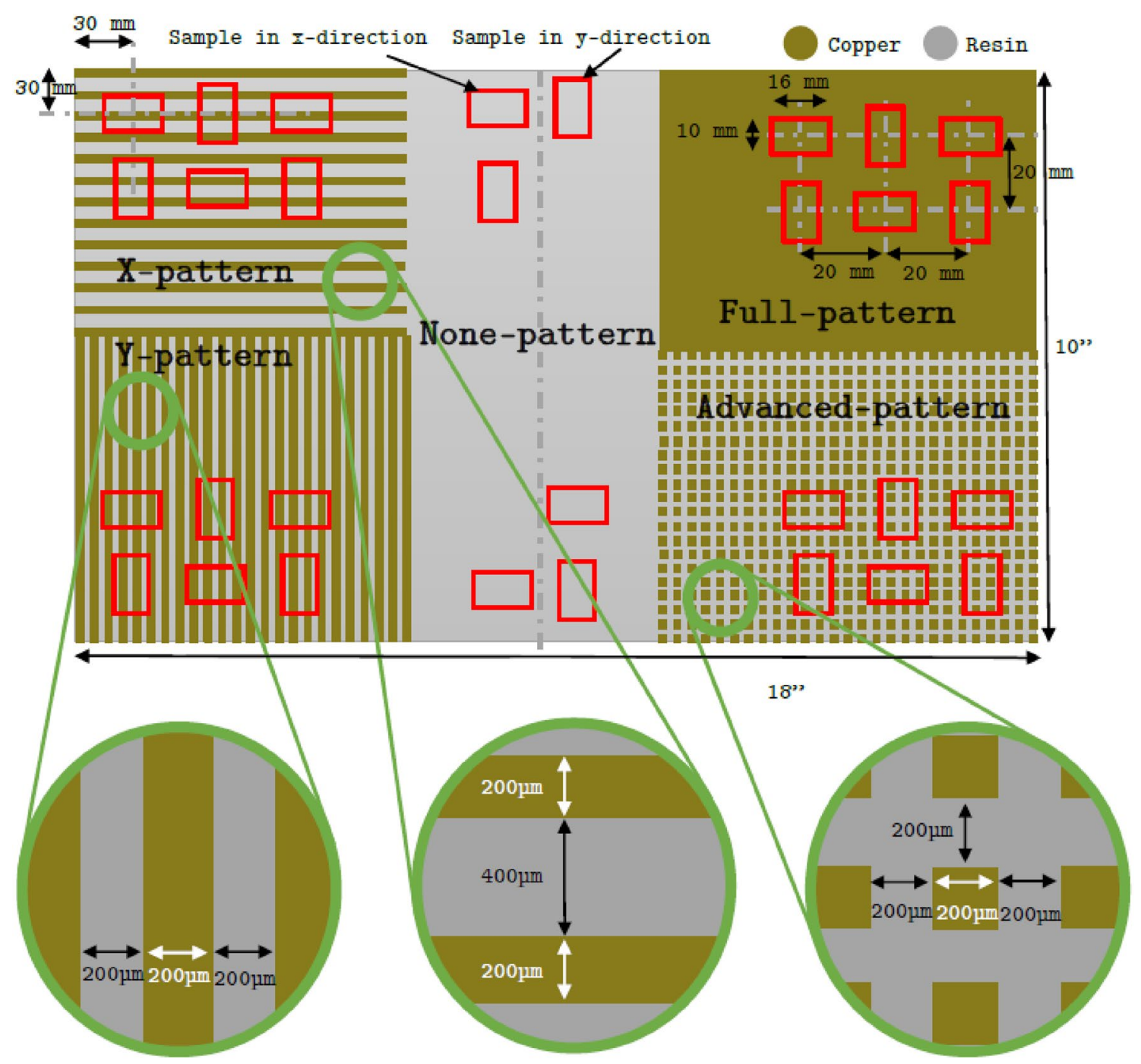

Fig. 5 Specially designed test board for copper pattern experiments including its dimensions. The $X-, Y$-, advanced, free of copper and full copper patterns are indicated. Furthermore, samples used for

squares, spaced on a 400 -by- $400 \mu \mathrm{m}$ grid. The area without copper is indicated by None-Pattern and the full copper area is labelled Full-Pattern.

Samples from this board were cut according to the red boxes indicated in Fig. 5 and the CTE-to-temperature relation was determined through a TMA. From identically produced boards, similar samples were cut, and used to make microsections and tensile test samples. The microsections were used for visual inspection, while the tensile test samples were used to determine the Young's Modulus of each type of copper pattern.

\subsection{Test board fabrication}

The copper patterns were fabricated by etching both sides, to ensure dimensional stability, of an Electro-Deposited
TMA, microsection and tensile testing in both $x$ - and $y$-directions are indicated by the red boxes. Please refer to the online version for the color representation of the figure

(ED) copper Nelco laminate (N4000-6FC [21]). Two identical boards were produced simultaneously side by side in a hot press. The build-up and as-designed layer thicknesses of the board are shown in Table 3. The Nelco laminate has a 2114 fiberglass cloth style in the middle. Two prepregs (Hitachi GEA-679F(J) [22]) with a 0106 fiberglass cloth style are pressed on both sides of the laminate to ensure proper epoxy resin flow and cure between the copper patterns. This produces, after curing, a multilayer board with uniform thickness, although the copper patterns vary locally. Besides protection, having cured epoxy between the copper patterns on the outsides is essential for transferring in-plane stresses through the entire build-up and not just the middle laminate in this case. Having epoxy resin between the copper features is also a minimum condition to determine the continuous layer properties using Eqs. 1-4. 
Table 3 Build-up of the test board with various ED-copper patterns

\begin{tabular}{lcl}
\hline Type of layer & Thickness $(\mu \mathrm{m})$ & $\begin{array}{l}\text { Fiberglass } \\
\text { cloth style }\end{array}$ \\
\hline Prepreg (Hitachi) & 54.1 & 0106 \\
ED-Copper patterns & 17.5 & \\
Laminate (Nelco) & 101.6 & 2114 \\
ED-Copper patterns & 17.5 & \\
Prepreg (Hitachi) & 54.1 & 0106 \\
\hline
\end{tabular}

The aforementioned U20MM modeler tool is able to predict thermoelastic material properties of the laminate and prepreg layers. Samples of copper-free areas are part of the fabricated test board to validate the micro-mechanics modeler. Samples of full copper areas were added for additional comparison and to determine the stiffness and CTE of ED copper, as this might be deviate from bulk copper properties.

Figure 6 shows the fabricated test board including a close-up of the $X$-pattern samples. Of each patterned area six TMA samples were produced, namely three in the $x$-direction and three in the $y$-direction. The TMA samples were milled and finally released by a single cut with a scalpel, as shown in Fig. 6(b). Multiple measurements in the same direction are done to check the consistency of the results. The $x$ - and $y$-directions are measured separately to determine the influence of the fiberglass cloth in both directions (i.e. the warp and fill directions) in the laminate and prepreg.

Two samples were taken of each patterned area to measure the Young's modulus: one in $x$-direction and one in $y$-direction. One measurement per sample was performed. The samples used for these measurements were taken from the board that was pressed on the right-hand side of the hot press. Samples taken from the board on the left-hand side of the hot press were used to make microsections.

The cut samples of each type of ED copper pattern in $x$ - and $y$-directions of the second board were molded into a polymer and polished to exhibit the inner microstructure of the cross section. Figure 7 shows one of the produced microsections of the advanced copper pattern in the $y$-direction including a close-up from which the layer thicknesses were determined. The resulting specimens show the inner-bonded structure of the laminate, copper layers and prepregs. The composition and thickness of the constituent layers were investigated and measured with the aid of a microscope and camera following IPC-TM-650 [23].

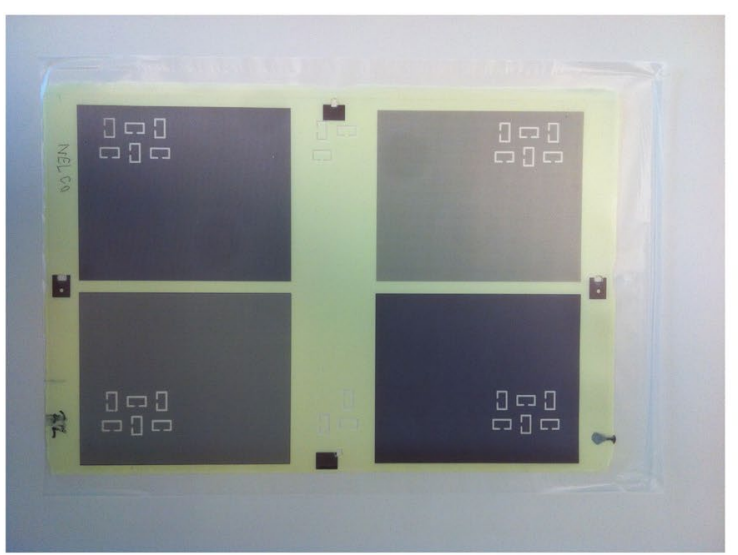

(a)

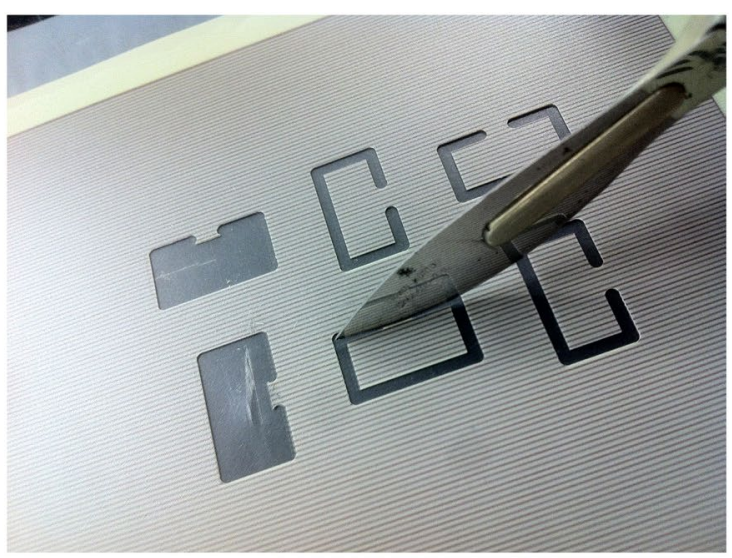

(b)

Fig. 6 Photographs of the board with the cut samples. a Four copper-patterned samples and copper free in the middle. b Close-up of the $X$-pattern samples

Fig. 7 Photograph of the microsection sample (the two white circles are the clamps) of the advanced copper pattern in $y$-direction and corresponding thickness measurement
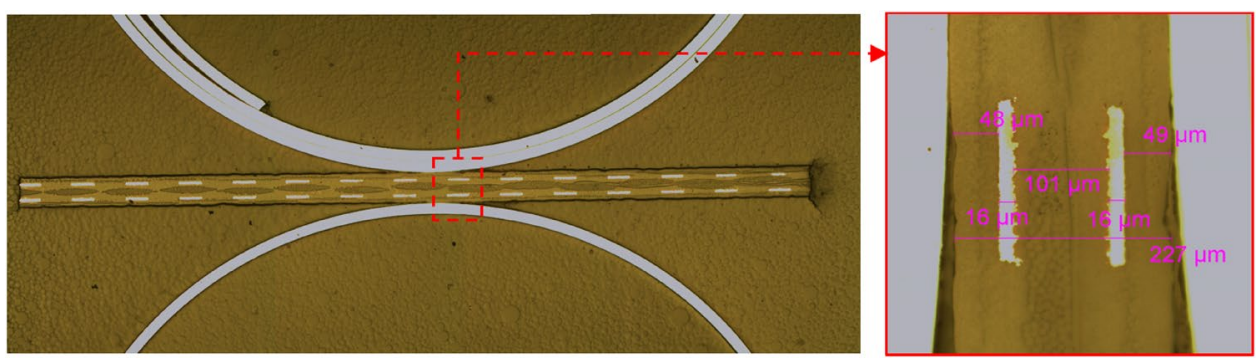


\subsection{Experimental results}

The experimental results from the TMA, microsections and tensile tests were used to determine the thermoelastic material properties for the patterned copper layers. Figure 8 shows the result of six TMA measurements for the $X$-pattern type in $x$-direction. During the TMA, the CTE is determined with respect to temperature. The figure shows the sample deformation due to heating following a userdefined time-temperature profile. From the response profile the glass transition temperature, $T_{g}$, and the CTE before and after glass transition can be determined. Also, the CTE during sample cooling can be determined.

The order of the successive measurements is indicated in red. The first measurement of a sample always shows the occurrence of an undulation in the expansion behavior near $T_{g}$ that can be explained by the release of stresses that are frozen-in during processing. When the sample is cooled down and retested (second measurement) a simple change in expansion rate is observed at $T_{g}$ indicating the sample is free of thermal stresses and other thermal history-related effects. This was confirmed by a third measurement, and again after reclamping and measuring on a second day (measurements
4-6). Note that in Fig. 8 measurements $1-3$ also used two heating cycles to collect more data, whereas for measurements 4-6 just a single heating cycle was used.

The undulation during each first measurement of a sample causes the CTE to vary; therefore, the CTE cannot be determined accurately during a first measurement. Also, non-fully cured material, moisture absorption and material re-alignment effects should be considered [24]. Hence, the first TMA measurement result is not used in this study. A summary of the TMA measurements is given in Table 4 . Each row lists the average results from the samples of that specific pattern type. The overall results show an average $T_{g}$ of $162.18{ }^{\circ} \mathrm{C}$, which is in agreement with the literature data for epoxy-resin boards (see Sect. 2.1).

The CTE below the glass transition temperature behaves expectedly and consistently with a relatively low standard deviation between measurements. The CTE values above the glass transition temperature behave unexpectedly, as they drop below the lowest CTE of the composite's constituents (see Table 2). The lower CTE values can be explained by internal shifting or sliding of both laminates or of individual glass fibers within the epoxy resin. In this paper, the CTE $>T_{g}$ is not utilized for characterizing the PCB

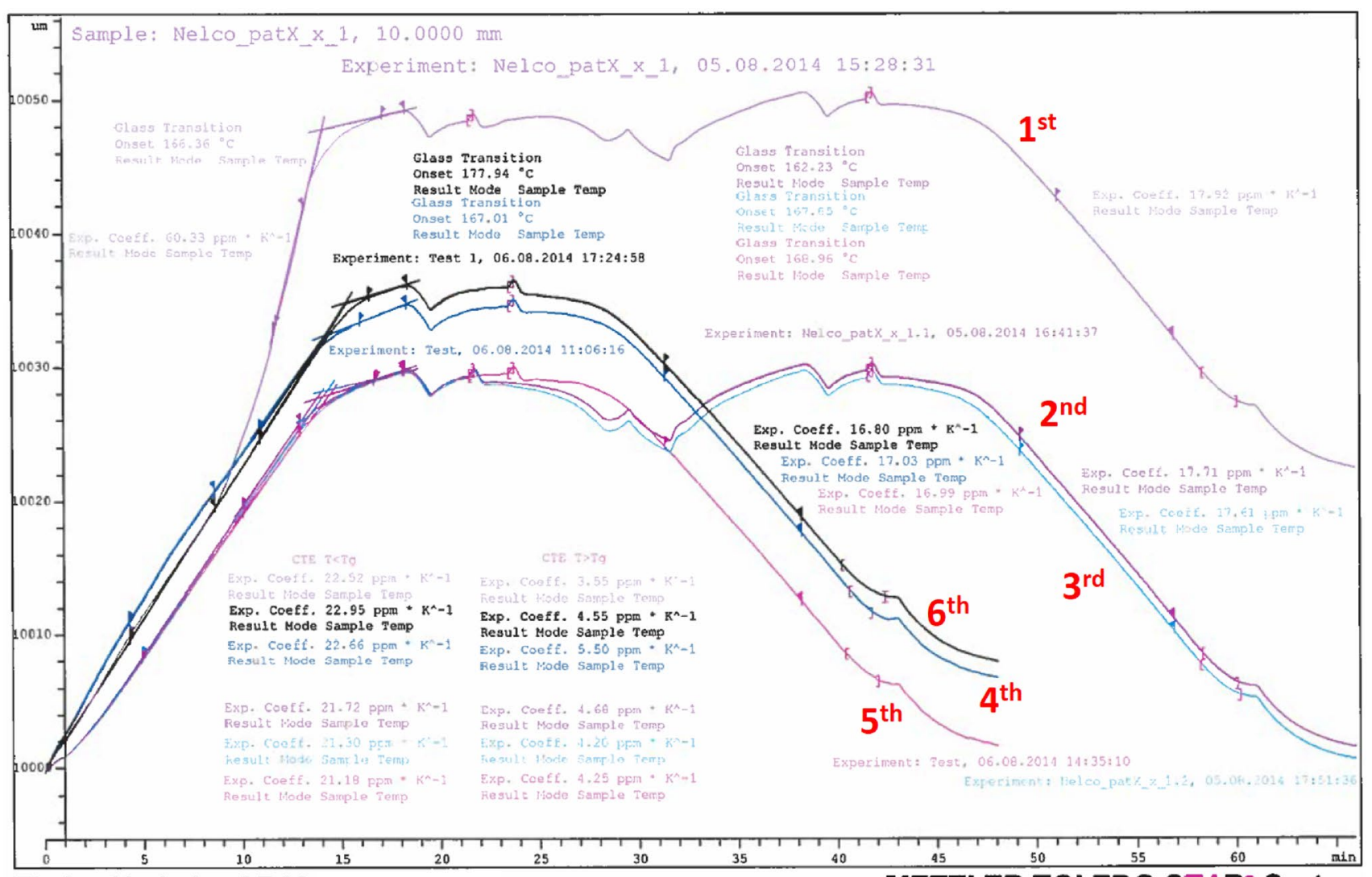

Thales Nederland B.V. METTLER TOLEDO STAR ${ }^{\mathrm{e}}$ System

Fig. 8 Six thermomechanical analysis (TMA) results of an $X$-patterned sample measured in $x$-direction 
Table 4 Summary of TMA results for each pattern type

\begin{tabular}{llllll}
\hline Pattern type in direction & $T_{g}\left({ }^{\circ} \mathrm{C}\right)$ & Std. dev. $T_{g}\left({ }^{\circ} \mathrm{C}\right)$ & $\begin{array}{l}\mathrm{CTE}<T_{g} \\
(\mathrm{ppm} / \mathrm{K})\end{array}$ & $\begin{array}{l}\mathrm{CTE}>T_{g} \\
(\mathrm{ppm} / \mathrm{K})\end{array}$ & $\begin{array}{l}\mathrm{CTE} \\
\text { cooling } \\
(\mathrm{ppm} / \mathrm{K})\end{array}$ \\
\hline$X$ in $x$ & 166.80 & 5.78 & 22.05 & 4.50 & 17.33 \\
$X$ in $y$ & 156.56 & 2.07 & 23.15 & 2.55 & 18.37 \\
$Y$ in $x$ & 156.80 & 1.61 & 23.45 & 2.73 & 19.13 \\
$Y$ in $y$ & 165.08 & 0.15 & 23.79 & 4.88 & 18.50 \\
Advanced in $x$ & 156.80 & 1.46 & 23.44 & 2.73 & 19.13 \\
Advanced in $y$ & 158.76 & 2.23 & 22.39 & 0.31 & 18.44 \\
Full in $x$ & 160.01 & 0.54 & 22.31 & 6.22 & 17.14 \\
Full in $y$ & 163.00 & 1.24 & 22.02 & 8.36 & 18.06 \\
None in $x$ & 157.70 & 0.35 & 22.81 & 3.15 & 18.45 \\
None in $y$ & 156.23 & 0.12 & 23.50 & 2.36 & 18.39 \\
\hline
\end{tabular}

constituent materials, but may serve as a reference for future work. For practical applications, in which most of the postprocessing temperatures are below the glass transition temperature, only the CTE $<T_{g}$ is used. Here, it is assumed that the effects of gelation and vitrification, which occur during the curing process [25], are captured within this CTE value.

Figure 9 shows the microsections in $x$ - and $y$-directions for all copper patterns. The copper is depicted in white, the $\mathrm{C}$-stage resin is colored light yellow and the outer contours are dark. The fiberglass reinforcement and its undulation are visible. Also, the difference in thickness between the thicker 2114 fiberglass cloth style in the middle and the thinner 0106 cloth styles surrounding the copper pattern can be observed. The copper patterns for the advanced patterns were designed to be $200 \mu \mathrm{m}$ with $200 \mu \mathrm{m}$ interspacing between. The final inner-board dimensions after lamination were determined from these microsections. A summary of the measured thicknesses is presented in Table 5 .

Table 5 shows the average thickness of the top and bottom prepreg layers, both copper-patterned layers, and the laminate in the middle. The average thickness was determined by measuring each sample at three locations along the cross section. The laminate in the middle is not influenced by the lamination process and remains at a similar thickness independent of the type of patterning. As the middle laminate is already cured, this behavior is expected. The ED copper layer becomes slightly thinner and this effect is also independent on the type of patterning. Finally, flattening of the prepreg layers has the most significant contribution to the total lay-up. This can be explained by the flow of resin between the copper patterns where possible. Also, some resin outflow at the board edges was observed.

During lamination, the space between the copper patterns is filled with resin from the prepreg layer. In the case of full copper patterning, the prepreg resin cannot flow between any copper. Similarly, for the None-pattern type, as the laminate is already cured, no resin flow into this layer is expected. As listed in Table 5, the average increase in layer thickness of the prepreg for those two pattern types is about $5 \mu \mathrm{m}$, which is $9 \%$. This increase in thickness is common for prepreg materials, in which $z$-direction expansion typically ranges around 7\% [22].

For the $X$-, $Y$ - and Advanced-pattern types, as shown in Fig. 5, the modeled copper fraction of that layer is $0.33,0.5$ and 0.25 , respectively. The expected thickness reduction of the prepreg layers with these pattern type can be determined by assuming complete filling of the open spaces in the copper pattern. To compute the resulting thickness of the prepreg layer the thickness reduction due to this flow of resin is taken into account, as well as the measured $9 \%$ z-direction expansion. The estimated thickness reduction for each pattern type is listed in Table 6. The estimated values correspond relatively well with the measured average values as listed in the last column, and repeated from Table 5. The systematic under estimation can be attributed to the copper layer that is etched on average $2.0 \mu \mathrm{m}$ (see Table 5) due to the lamination pretreatment process and minor resin outflow at the edges of the test board.

It was confirmed that single prepreg layers increase in thickness if they reside between full laminates or copper layers due to $z$-direction expansion according to supplier specifications. If a prepreg layer is adjacent to a copper layer with features, the amount of resin volume flow from the prepreg layer between the copper features is equal to the volume of removed copper from the adjacent layer. The $z$-direction expansion of prepreg layer is not insignificant and must be taken into account. Overall, this results in a thickness reduction of the entire prepreg layer, as the void volume is larger than the volume attributed to the $z$-direction expansion. 


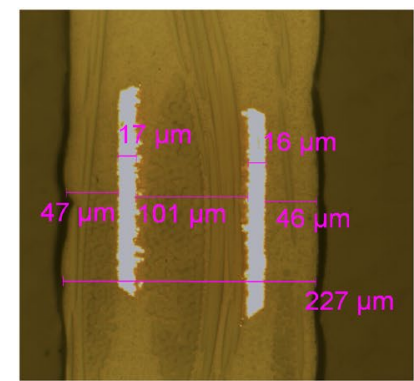

$x$ in $x$

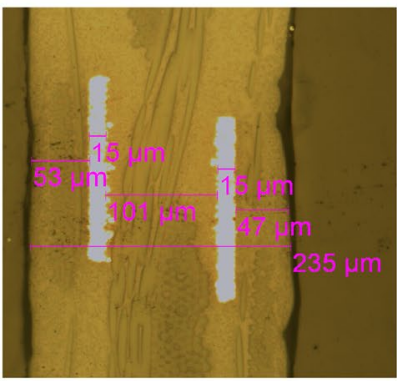

Advanced in $x$

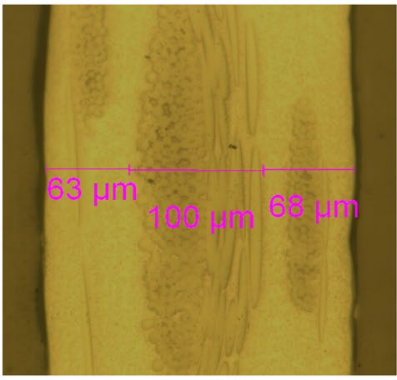

None in $x$

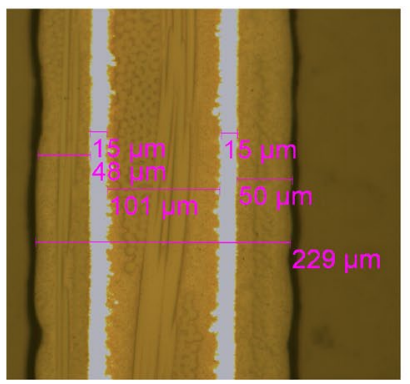

$x$ in $y$

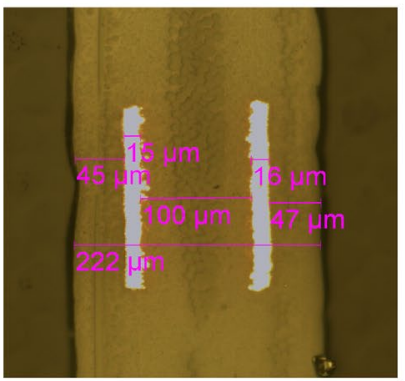

Advanced in $y$

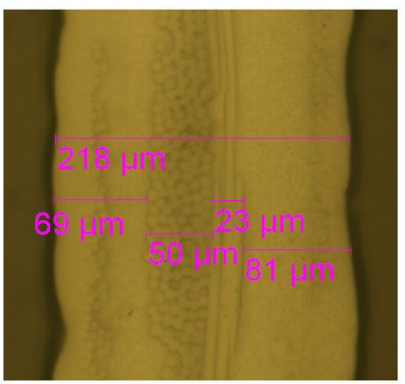

None in $y$

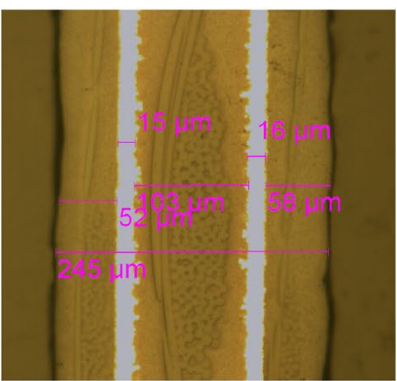

$Y$ in $x$

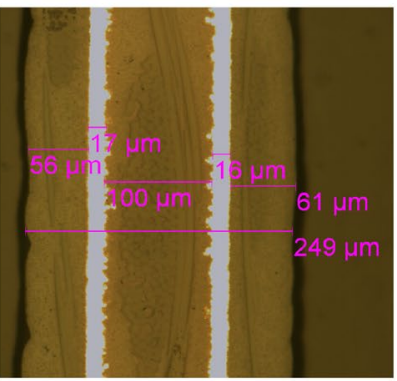

Full in $x$

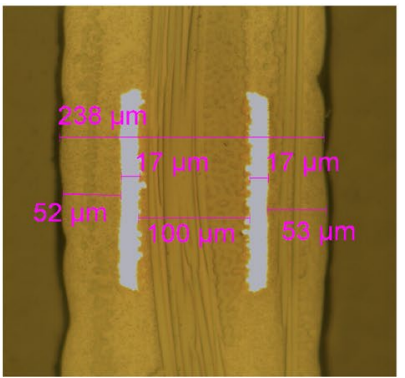

$Y$ in $y$

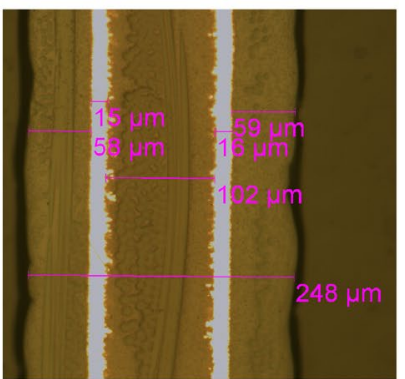

Full in $y$

Fig. 9 Microsections in $x$ - and $y$-directions for all copper patterns and corresponding thickness measurement

Table 5 Average thickness measurements after lamination of the copper patterns microsections; both the prepreg and copper layers are the average of the top and bottom layers, respectively

\begin{tabular}{lllll}
\hline Pattern type in direction & $\begin{array}{l}\text { Prepreg layers } \\
(\mu \mathrm{m})\end{array}$ & $\begin{array}{l}\text { Copper layer } \\
(\mu \mathrm{m})\end{array}$ & Laminate $(\mu \mathrm{m})$ & Total lay-up $(\mu \mathrm{m})$ \\
\hline As-designed (Table 3) & 54.1 & 17.5 & 101.6 & 244.8 \\
$X$ in $x$ & 48.4 & 15.5 & 101.3 & 227.2 \\
$X$ in $y$ & 49.4 & 15.2 & 101.0 & 229.3 \\
$Y$ in $x$ & 53.4 & 15.5 & 103.0 & 241.7 \\
$Y$ in $y$ & 52.5 & 15.7 & 102.3 & 234.3 \\
Advanced in $x$ & 49.7 & 15.5 & 101.0 & 234.3 \\
Advanced in $y$ & 47.8 & 15.7 & 100.7 & 224.3 \\
Full in $x$ & 58.4 & 16.0 & 100.7 & 247.3 \\
Full in $y$ & 58.5 & 15.4 & 102.7 & 247.0 \\
None in $x^{\mathrm{a}}$ & 61.7 & - & 101.6 & 225.0 \\
None in $y^{\mathrm{a}}$ & 57.7 & - & 101.6 & 217.0 \\
\hline
\end{tabular}

${ }^{\text {a }}$ To determine these values the interfaces between the fiberglass cloths were used, which are less accurate to determine 
Table 6 Estimated thickness of the prepreg layers after lamination

\begin{tabular}{lllll}
\hline Pattern type & $\begin{array}{l}\text { Copper } \\
\text { fraction } \\
(-)\end{array}$ & $\begin{array}{l}\text { Computed } \\
\text { thickness } \\
\text { reduction } \\
(\mu \mathrm{m})\end{array}$ & $\begin{array}{l}\text { Estimated } \\
\text { thickness } \\
(\mu \mathrm{m})\end{array}$ & $\begin{array}{l}\text { Measured avg. } \\
\text { thickness }(\mu \mathrm{m})\end{array}$ \\
\hline$X$ & 0.33 & 11.6 & 47.4 & 48.9 \\
$Y$ & 0.5 & 8.8 & 50.2 & 52.9 \\
Advanced & 0.25 & 13.1 & 45.9 & 48.8 \\
\hline
\end{tabular}

\section{Thermoelastic material properties for prepreg and laminate layers}

\subsection{Properties of the prepreg and laminate layers}

The measured thickness and the fiberglass volume of the prepreg and laminate layers can be determined, which are then used to compute the thermoelastic properties of that layer. The fiber volume fraction, $V_{f}$, was determined by assuming that the fiberglass volume remains constant and all deviations in board height are accounted for by the resin flow and expansion. The computed thermoelastic properties of the prepreg (0106) and laminate (2114) are presented in Fig. 10 as a function of the fiber volume fraction. The micro-mechanics modeler is used to compute the CTE, see Fig. 10(a), and Young's modulus, see Fig. 10(b), for a number of fiber volume fractions indicated by the plot markers; the lines are interpolated thereafter. For the laminate both the principle $x$ - and $y$-directions are computed, although they do not differ significantly. The weave properties of the layer specific weaves, the 0106 and 2114 cloth styles, are taken as listed in Table 1, and the material properties of the epoxy resin and glass fiber are taken as

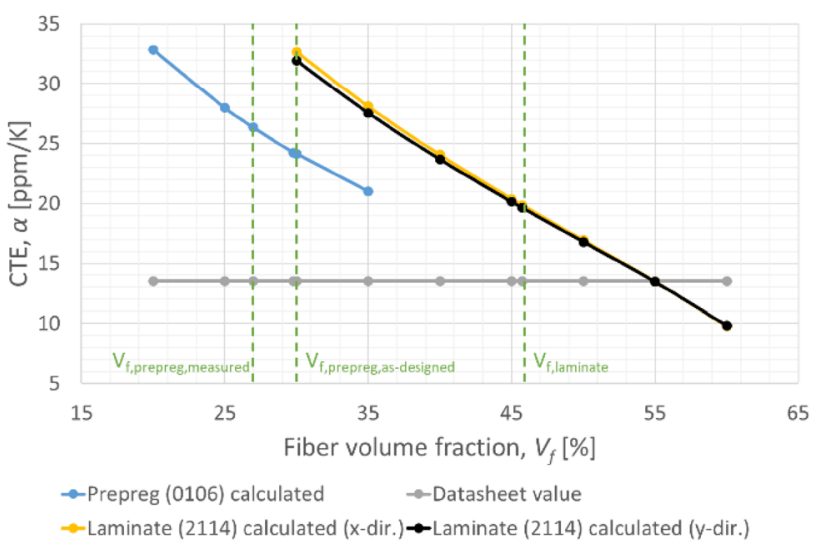

(a)

Fig. 10 Effect of fiber volume fraction for epoxy prepreg (0106) and laminate (2114) composites in $x$-direction and $y$-direction (2114 style) as determined by the micro-mechanics modeler. The reference listed in Table 2. Figure 10 also depicts the (constant) reference properties as listed by material datasheets [21, 22].

Figure 10 demonstrates that prepreg layers with the same fiberglass cloth style but different thicknesses have a CTE and Young's modulus that is dependent on the fiber volume fraction. The fiber volume fraction of the prepreg with asdesigned thickness is computed to be $29.8 \%$ indicated by the middle vertical dashed line. With the measured thickness of the None-pattern, the fiber volume fraction was lower $(27.0 \%)$ due to the prepreg expansion. The laminate has a much higher fiber volume fraction of $45.7 \%$. It has a different fiberglass cloth style compared to the prepreg, so the relationship between the fiber volume fraction, and the CTE and Young's modulus is different, as shown in Fig. 10. Datasheets do not take this relationship into account and are thus constant for all fiber volume fractions. The 0106 fiberglass cloth style has equal properties in $x$ - and $y$-directions, and hence the entire prepreg behaves symmetrical in $x$ - and $y$-directions.

From this, it can be concluded that there is a difference in fiber volume fraction between as-designed and measured layers. A deviation in fiber volume fraction may result in a significant change in material properties. Therefore, it is important to use the fiber volume fraction and a micromechanics modeler to compute the right material properties in terms of CTE and Young's modulus of each layer.

\subsection{CTE validation at the macroscopic level}

Using the none-patterned samples the CTE is validated. The CTE of each constituent layer is determined as shown in the previous section. The CTE of the fabricated none-patterned samples, as was shown in Fig. 5, can be determined

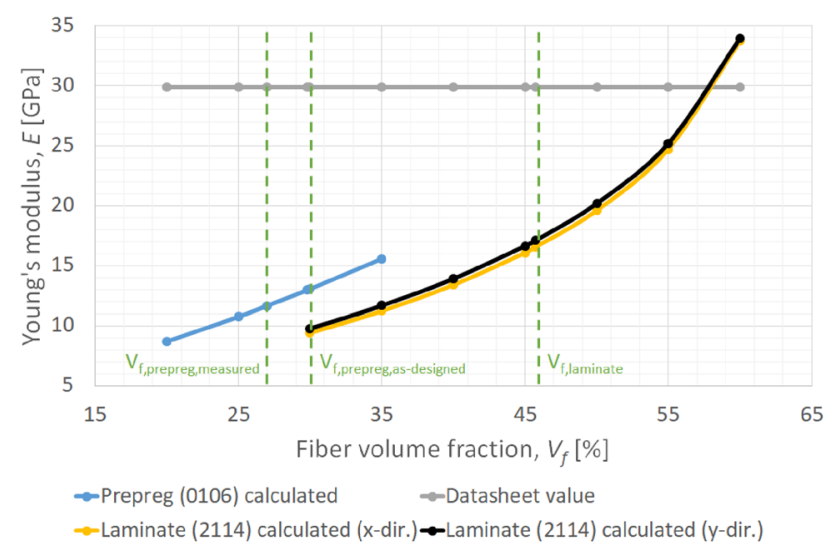

(b)

properties of the datasheets are also shown. Please refer to the online version for the color representation of the figure. a Effect on CTE. b Effect on Young's modulus 
assuming the prepreg and laminate are position in parallel according to Eq. 2. Both the as-designed and measured thicknesses of the prepreg layer are used as input for the micro-mechanics modeler.

Table 7 lists the results of the TMA measurements and compares them to the computed values with an as-designed and measured prepreg thickness. The last column lists the CTE as can be determined from the datasheet material properties using the as-designed thickness.

According to Table 7, the new material model differs $0.8 \mathrm{ppm} / \mathrm{K}$ in $x$-direction and $1.8 \mathrm{ppm} / \mathrm{K}$ in $y$-direction compared to the TMA results for the as-designed thickness. When the measured thickness is used as input for the micro-mechanics modeler, the deviation even diminishes further. Both show a significant increase in accuracy compared to the datasheet values, which are more than $1.7 \times$ lower than the TMA results. This demonstrates the necessity of using a fiber fraction-dependent multilayer board model.

\subsection{Young's modulus validation at the macroscopic level}

Using the copper-patterned samples, the Young's modulus at the macroscopic multilayer level is determined experimentally. Tensile testing was done according to NEN-EN 658-1 [26], which specifies the conditions for determination of tensile properties of ceramic matrix composite materials with continuous fiber reinforcement at ambient temperature. Table 8 lists the measured values for the different patterning types and directions of tensile testing.

As is to be expected, samples with the most copper have the highest Young's modulus. Furthermore, the Young's modulus for the $X$-, $Y$ - and Advanced-pattern types are between the full and none copper pattern. An overall observation is that the measurements in $y$-direction are on average about $0.84 \mathrm{GPa}$ higher than in $x$-direction. As the patterning of the full and none-patterned samples has no influence on the direction, the deviation between both directions can be attributed to the reinforcement of the 2114 fiberglass cloth style, which is stiffer in the $y$-direction due
Table 8 Young's modulus measurements of fabricated copper-patterned samples

\begin{tabular}{lll}
\hline Pattern type & Measured direction & $\begin{array}{l}\text { Young's } \\
\text { modulus } \\
(\mathrm{GPa})\end{array}$ \\
\hline$X$ & $x$ & 17.7 \\
& $y$ & 19.0 \\
$Y$ & $x$ & 18.7 \\
& $y$ & 19.7 \\
Advanced & $x$ & 17.9 \\
\multirow{2}{*}{ Full } & $y$ & 18.1 \\
& $x$ & 20.4 \\
None & $y$ & 21.6 \\
& $x$ & 17.0 \\
\end{tabular}

to higher number of glass fibers in the $y$-direction. The average difference in Young's modulus between the $x$ - and $y$-directions for these two pattern types is $0.86 \mathrm{GPa}$. The micro-mechanics modeler predicts an increase in Young's modulus in the $y$-direction of $0.60 \mathrm{GPa}$, which is close to the average measured value.

Using the micro-mechanics modeler the Young's modulus for each constituent layer of the none-patterned sample can be determined. Following Eq. (2), the Young's modulus of the multilayer sample can be determined. For the $x$ - and $y$-directions a Young's modulus of 13.5 and $14.2 \mathrm{GPa}$ is computed, respectively, which, compared to Table 8 , is a slight under prediction. This underestimation will cause an overestimation of the layers with copper patterning, as will be discussed in Sect. 6. Using datasheet values (see Fig. 10) the Young's modulus in $x$ - and $y$-directions are 29 and 25 $\mathrm{GPa}$, respectively, which is more than $1.5 \times$ higher as the measured values. Again, showing the necessity of using a fiber fraction dependent multilayer board model.

From Table 8 it can also be concluded that the type of copper patterning has minimal influence on the Young's modulus at the macroscopic level. The Young's modulus at this level is dominated by the fiberglass cloth.
Table 7 CTE in $x$ - and $y$-directions from TMA measurements, micromechanics modeler and datasheet values for the fabricated none-patterned sample

\begin{tabular}{|c|c|c|c|c|c|c|c|}
\hline & \multirow[t]{3}{*}{ TMA } & \multicolumn{4}{|c|}{ Micro-mechanics modeler } & \multirow{2}{*}{\multicolumn{2}{|c|}{ From datasheets }} \\
\hline & & \multicolumn{2}{|c|}{ As-designed thickness } & \multicolumn{2}{|c|}{ Measured thickness } & & \\
\hline & & & $\begin{array}{l}\text { TMA devia- } \\
\text { tion }\end{array}$ & & $\begin{array}{l}\text { TMA devia- } \\
\text { tion }\end{array}$ & & $\begin{array}{l}\text { TMA } \\
\text { devia- } \\
\text { tion }\end{array}$ \\
\hline $\mathrm{CTE}_{x}(\mathrm{ppm} / \mathrm{K})$ & 22.8 & 21.9 & 0.8 & 22.8 & 0.0 & 13.3 & 9.4 \\
\hline $\mathrm{CTE}_{y}(\mathrm{ppm} / \mathrm{K})$ & 23.5 & 21.7 & 1.8 & 22.6 & 0.9 & 13.4 & 10.1 \\
\hline
\end{tabular}


Fig. 11 Scheme of the method to determine copper-patterned layer properties

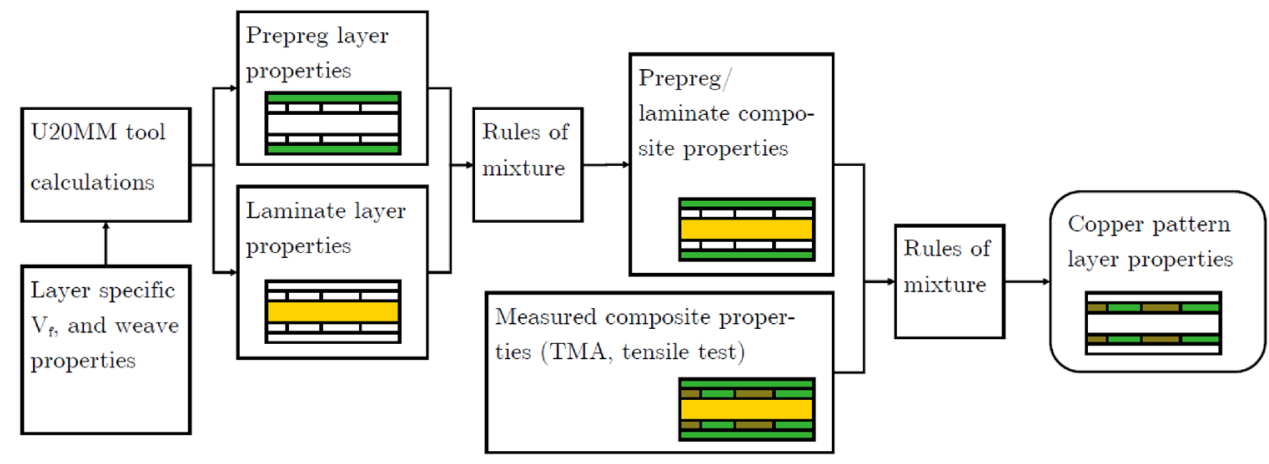

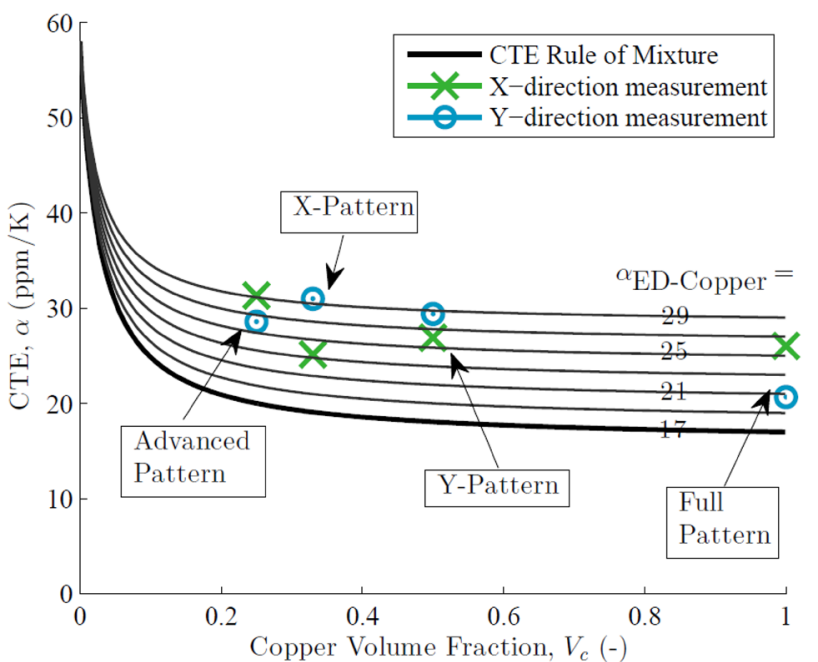

Fig. 13 CTE of patterned-copper layers as a function of the copper volume fraction. The computed CTE for various ED copper CTEs is also depicted

Following this scheme, Fig. 12 shows the deduced Young's modulus for each type of copper-patterning in $x$ - and $y$-directions, indicated by $\mathrm{X}$ (green) and $\mathrm{O}$ (blue), respectively. The figure shows that there is a linear correlation between the copper volume fraction, $V_{c}$, and the Young's modulus. The inverse and advanced rules of mixture, Eqs. 3 and 4, respectively, are not representative to determine the properties of a copper-patterned layer. The parallel rule of mixture (Eq. 1) however correlates well with the measured values. This can be explained by the fact that the inverse and advanced rules of mixture assume iso-stress conditions, which is not the case for the parallel rule of mixture that is based on iso-strain conditions. Similar to what is assumed in CLT, the adhesion forces of the cured resin in a fabricated multilayer board do not allow the constituent layers to shear resulting in iso-strain conditions.

The results for the full-copper patterning show a Young's Modulus of about $120 \mathrm{GPa}$, which is consistent with reported literature values (100-135 GPa) for thick ED copper [27]. For the $X-, Y$ - and Advanced-pattern types, the 
Young's modulus is higher than predicted by the rule of mixture. This overshoot is due to the fact that the micromechanics modeler underestimates the Young's modulus of the constituent layers, as discussed in Sect. 5.3.

Based on iso-strain conditions, the CTE of the copper patterning can be determined as a function of the copper volume fraction according to Eq. 2. Figure 13 presents the computed CTE values of the copper-patterned layers. The black lines indicate the layer's CTE computed using various CTE values (viz. 17-29 ppm/K) of the ED copper, as the exact CTE value is unknown due to the variance of the plating process and the fact that the ED copper-plated layer is relatively thin $[28,29]$. As shown in the figure, the measured CTE values for all types of copper patterning lie within the computed range. Also, the predicted trend by the rule of mixture is followed by the measurement results. In the case of full-copper patterning, the CTE is lower than other patterning types, corresponding to the fact that the CTE of copper is lower than that of the resin.

\section{Conclusions}

A method to determine the thermoelastic material properties for cured prepreg and laminate, with and without copper patterning, has been presented. Using a micro-mechanics modeler the constituent non-copper layer properties are determined. By assembling these layer properties and comparing them to fabricated multilayer boards that include copper pattering, the properties of copper patterning can be deduced, see Fig. 11. The presented model increases the accuracy for determining the impact that constituent layers have on the multilayer board significantly in comparison to the use of common datasheet material properties.

Following to the presented model, the Young's modulus and CTE of various types of copper patterning have been determined experimentally. Patterned-copper layer properties, Young's modulus and CTE have been determined for layers with continuous copper tracks in both longitudinal and parallel directions, and for an advanced copper pattern. The results show that voids between copper patterns are filled by the flow of resin; however, a $z$-direction expansion of prepreg being cured of 7-9\% must also be taken into account.

This research has shown that the thermoelastic material properties of cured prepreg and laminate layers are dependent on the material properties of the constituents, the type of fiberglass reinforcement weave and most importantly the fiber volume fraction. Depending on these properties, the Young's modulus and CTE varies from 11 to $31 \mathrm{GPa}$ and from 10 to $28 \mathrm{ppm} / \mathrm{K}$, respectively. Datasheet values do not take fiber volume fraction into account and therefore predict a Young's modulus $1.5 \times$ too high and a CTE $1.7 \times$ too low.

Experiments have shown that the constituents and layup of the entire board contribute to the macroscopic multilayer properties. Experiments in which the measurement direction is varied show that the fiberglass reinforcement is a dominant factor in determining the macroscopic multilayer board properties. Experiments have also shown that the fabricated multilayer board follows iso-strain conditions. Hence, material properties depend linearly on the volume fraction of copper and follow the rule of mixtures (Eq. 1) independent of the type of patterning.

Altogether, this offers PCB material suppliers a more accurate method to supply multilayer material properties. Moreover, as material properties show large variation depending on the fiber volume fraction and copper volume fraction, this method offers the possibility to predict and model PCB designs with respect to bow and twist, reliability, asymmetry, and many more aspect.

Acknowledgements The authors would like to thank the Thales Group Competence Center - Printed Circuit Boards (GCC-PCB) located at Thales Nederland B.V. for making this research possible and for providing the required equipment, in-depth discussions about the theory and applications, and financial support.

Funding Funding to publish this work Open Access was provided by VSNU Vereniging van Universiteiten.

Open Access This article is distributed under the terms of the Creative Commons Attribution 4.0 International License (http://creativecommons.org/licenses/by/4.0/), which permits unrestricted use, distribution, and reproduction in any medium, provided you give appropriate credit to the original author(s) and the source, provide a link to the Creative Commons license, and indicate if changes were made.

\section{References}

1. IPC, International Technology Roadmap for Electronic Interconnections (2015)

2. IPC, PCB Technology Trends (2016)

3. R.R. Tummala, E.J. Rymaszewski, A.G. Klopfenstein, Microelectronics Packaging Handbook-Technology Drivers Part I (1997)

4. G. Dudnikov, North American PCB Military Technology Roadmap (IPC Technology Interchange, Washington, D.C., 2009)

5. M. Slomp, Deformation of Multi-layer Printed Circuit Boards (Enschede, 2012)

6. G.A. Schuerink, M. Slomp, W.W. Wits, R. Legtenberg, E.A. Kappel, Modeling printed circuit board curvature in relation to manufacturing process steps. Proced. CIRP 9, 55-60 (2013)

7. H. Guojun, G.K. Yong, L. Jing-en, L.W. Chin, X. Baraton, Thermoelastic properties of printed circuit boards: effect of copper trace. in Microelectronics and Packaging Conference, 2009. EMPC 2009. European (2009), pp. 1-6 
8. R. De Vries, E. Lamers, S. Wijskamp, B.V. Rodriguez, R. Akkerman, Theoretical Background U20MM Modeler (University of Twente, Enschede, 2005)

9. C.F. Coombs Jr., H. Holden, Printed Circuits Handbook, 7th edn (McGraw-Hill, New York, 2016)

10. J. Varteresian, Fabricating Printed Circuit Boards (Elsevier, New York, 2002)

11. M.W. Jawitz, M.J. Jawitz, Materials for Rigid and Flexible Printed Wiring Boards (CRC Press, Boca Raton, 2006)

12. A.C. Loos, G.S. Springer, Curing of epoxy matrix composites. in Engineering Mechanics of Fibre Reinforced Polymers and Composite Structures (Springer, Vienna, 1994), pp. 263-285

13. S.M. Sabzevari, Cure kinetics and process modeling of a carbonfiber thermoplastic-toughened epoxy resin prepreg. $\mathrm{PhD}$ Thesis, Wichita State University, 2010

14. M. Tarr, Printed Circuit Boards-Alternative Board Materials (University of Bolton, Bolton, 2014)

15. S.A. Hussain, B.S. Reddy, V.N. Reddy, Prediction of elastic properties of FRP composite lamina for longitudinal loading. ARPN J. Eng. Appl. Sci. 3, 70-75 (2008)

16. J. Loyer, R. Kunze, X. Ye, Fiber weave effect: Practical impact analysis and mitigation strategies. DesignCon. (2007)

17. N.R. Sottos, J.M. Ockers, M. Swindeman, Thermoelastic properties of plain weave composites for multilayer circuit board applications. J. Electron. Packag. 121(1), 37-43 (1999)

18. R.M. Jones, Mechanics of Composite Materials (Bull Ridge Publishing, Blacksburg, 1999)

19. R. De Vries, E. Lamers, S. Wijskamp, B.V. Rodriguez, R. Akkerman, U20MM micromechanics modeler software tool. University of Twente, 2005. [Online]. https://www.utwente.nl/en/et/ms3/ research-chairs/pt/research/research-themes/tools. Accessed 28 August 2017

20. R. Warnet, Akkerman, Composites Course Reader (2010)

21. Park Electrochemical Corp., Nelco® N4000-6 FC, Rev 6-06 ed

22. Hitachi, $M C L-E-679 F(J)$ (2013)

23. IPC, 2.1.1 Microsectioning, Manual and Semi or Automatic. in IPC-TM-650 Test Methods Manual (IPC, 2015), p. 8

24. J. Foreman, S.R. Sauerbrunn, C.L. Marcozzi, Exploring the sensitivity of thermal analysis techniques to the glass transition. TA Instruments: Applications Library Search [online] (2006)

25. G. Twigg, Master Thesis: tool-part interaction in composites processing (2001)

26. NEN, Determination of tensile properties. in NEN-EN 658-1: Advanced Technical Ceramics-Mechanical Properties of Ceramic Composites at Room Temperature (1998), p. 19

27. H. Miura, K. Suzuki, K. Tamakawa, Fluctuation mechanism of mechanical properties of electroplated-copper thin films used for three dimensional electronic modules. International Conference on Thermal, Mechanical and Multi-Physics Simulation Experiments in Microelectronics and Micro-Systems, 2007. EuroSime 2007, pp. 1-6 (2007)

28. A. Volinsky, J. Vella, I. Adhihetty, V. Sarihan, L. Mercado, B. Yeung, W. Gerberich, Microstructure and Mechanical Properties of Electroplated $\mathrm{Cu}$ Thin Films. in MRS Proceedings, vol. 649 (2000)

29. Y. Xiang, X. Chen, J. Vlassak, The Mechanical Properties of Electroplated $\mathrm{Cu}$ Thin Films Measured by means of the Bulge Test Technique. in MRS Proceedings, vol. 695 (2001) 